\title{
Biology and infection mechanisms of Cryptosporiopsis spp fungus causing blight disease on cashew (Anacardium Occidentale L.)
}

\author{
Menge Dominic ${ }^{1,}$, Martha Makobe ${ }^{2}$, Bonaventure Agboton ${ }^{3}$, Shamte Shomari $^{4}$, \\ Andreas Tiedemann ${ }^{5}$ \\ ${ }^{1}$ Biological Sciences Department, Maasai Mara University; P. O. Box 861 Narok, Kenya \\ ${ }^{2}$ Department of Botany, Jomo Kenyatta University of Agriculture and Technology, Nairobi, Kenya \\ ${ }^{3}$ International Centre of Insect Physiology and Ecology (ICIPE); P. O. Box 30772-00100 Nairobi, Kenya \\ ${ }^{4}$ Cashew Research Programme, Naliendele Agricultural Research Institute (NARI), P.O. Box 509, Mtwara, Tanzania \\ ${ }^{5}$ University of Göttingen, Grisebachstrasse 6, 37077 Göttingen, Germany
}

\section{Email address:}

dominicmenge@yahoo.co.uk (M. Dominic)

\section{To cite this article:}

Menge Dominic, Martha Makobe, Bonaventure Agboton, Shamte Shomari, Andreas Tiedemann. Biology and Infection Mechanisms of Cryptosporiopsis spp Fungus Causing Blight Disease on Cashew (Anacardium Occidentale L.). Journal of Plant Sciences.

Vol. 2, No. 6, 2014, pp. 266-275. doi: 10.11648/j.jps.20140206.12

\begin{abstract}
Efficient isolation, purification and identification techniques of a new pathogenic fungus of cashew were developed. In the study, aspects related to identification, characterization of conidial morphology and infection of the Cryptosporiopsis spp fungus were investigated. Macroscopic observations of symptoms and signs of cashew blight disease as well as isolation, culturing and morphological characterization of its causative agent were carried out. Cryptosporiopsis spp fungus penetration and infection mechanisms on susceptible cashew leaves were studied. Cashew leaves were inoculated with fungal suspensions by pricking with pin, clipping with scissors and painting a fungal suspension onto the leaves with a paintbrush. Pathogenecity assays were performed on cashew leaves, nuts and apples. Irregular spot lesions, with a brown margin, formed on leaves especially on the young leaves. Direct conidial transfer was found to be the efficient method of isolation because it was less prone to contamination. Blight macroconidia are typical of Cryptosporiopsis having ellipsoidal, rounded at the apex, tapering into a scar at the base. Stomatal penetration of Cryptosporiopsis spp was visible through the simple staining technique using lactophenol solution. Penetration was by germinating conidia through a cuticle that caused an infection in leaves. Paint brush method of inoculation was found to be more effective as compared to other two methods based on the lesion size. Leaves inoculated with Cryptosporiopsis spp isolate began curling after $72 \mathrm{~h}$ thereafter developed dark, irregularly shaped spots with brown margins. An increased understanding of cashew blight is important for developing strategies for its management.
\end{abstract}

Keywords: Cryptosporiopsis spp, Macroscopic, Penetration, Cashew

\section{Introduction}

Cashew (Anacardium Occidentale L.) has a wide range of applications therefore a high demand across the world (Kannan et al., 2009). However, an attack from diseases has remained an impediment to the supply of quality cashew products. Some diseases attacking cashew include inflorescence blight caused by Lasiodiplodia theobromae and powdery mildew Noack caused by Oidium anacardii. Three fungal genera Lasiodiplodia, Fusarium and Pestalotia are associated with infected twigs and diseased inflorescences in cashew (Adeniyi et al., 2011). Cashew leaf and nut pathogen was first described from Tanzania by Sijaona (2006) and elaborated by Global Plant Clinic (GPC). Diseased plant materials were sent to GPC October 2003, where a new and undescribed Cryptosporiopsis $s p$ was consistently isolated from the nut and leaf lesions. One isolate (Cryptosporiopsis sp. IMI391611) has been deposited to the CABI Bioscience Culture Collection. The GPC received another cashew inquiry from the Caribbean in 2007 which on further investigation proved to be exactly the same fungus and disease found in 
Tanzania. The pathogen is being characterized further into its taxonomical nomenclature (GPC, 2010).

Infected cashew leaves develop silver/grey lesions with a dark reddish brown margin that enlarge and coalesce causing defoliation (Sijaona et al., 2006). Infected young nuts blacken while older nuts results in characteristic dark lesions that under favorable conditions white spore masses of the fungus were observed within the nut lesions. The disease has been shown to be most active during wet weather especially during off-season rains, where severe infections affect the young flushing material (Sijaona et al., 2006). Anacardiaceae Lindl., the cashew family, consist about 82 genera and over 700 species, the genus Anacardium contains 8 species which are native to the north eastern Brazil (Azam-Ali and Judge, 2000). Cashew nuts are highly nutritious containing $21.70 \%$ protein and $47.20 \%$ fat and the mineral composition contained in cashew apples is useful for human health (Marc et al., 2011; Umeh, 2007; Ogunwolu et al., 2010). Cashew nut tree provides food, employment, income and the wood is used for carpentry mainly in the construction of boats and ferries while other products derived from it include firewood and charcoal (Akinwale, 2000). Insect repellents, natural insecticides and medicinal products have been manufactured from cashew resins (Cavalcante et al., 2003). Therefore the objectives of this study included isolation, purification and identification of pathogenic fungus causing leaf and nut blight disease of cashew. Its ultimate goal is to update previous information about the pathogen and the disease it causes, to present outcomes of studies on conidial morphology and in vitro cultural studies mechanisms of penetration and in situ progression of the disease.

\section{Materials and Methods}

\subsection{Symptomatic Characterization, Isolation and Identification of Cryptosporiopsis spp Causing Cashew Blight}

Cashew plantations were regularly inspected for disease in many locations across Tanzania, during the period 2010-2011. Susceptible cashew clone AC 4 was used throughout the experiment. Macroscopic appearances of signs and symptoms of leaf and nut blight disease from the adaxial and abaxial leaf surfaces of cashew (Anacardium occidentale L.) were captured with a sharply focused digital camera. An experiment to identify an easy and fast method of isolation of Cryptosporiopsis spp was conducted using the recommended media, Potato dextrose agar (PDA) at Naliendele Agricultural Research Institute, Tanzania. Samples of cashew leaves were collected from various locations, kept in plastic bags and transferred to the lab according to Ammar and El-Naggar, 2011. Individual samples were cut into small pieces $0.5 \mathrm{~cm}$, surface sterilized for $2 \mathrm{~min}$ in a sodium hypochlorite solution $(3.85 \mathrm{~m} / \mathrm{v})$ washed three times and placed on filter papers in petri dishes.

The three methods of isolation tested were direct conidial transfer, conidial suspension transfer and transfer of lesion from advancing margin. A 6-mm diameter lesion with profuse sporulation was taken and aseptically slided at four lines running across the diameter of $9 \mathrm{~cm}$ diameter sterilized Petri dish for direct conidial transfer. A conidial suspension of $10^{6}$ $\mathrm{mL}^{-1}$ was uniformly spread over the surface of the media for conidial suspension transfer. In lesion transfer, four lesions with complete black sporulating appearance were aseptically put at four places within the Petri dish. The experiment was carried out in a completely randomized design with three treatments replicated twenty times. Evaluation of growth and contamination was carried out on each Petri dish $72 \mathrm{~h}$ after isolation following the scaling in table 1. Infected leaf, pseudo fruit and nuts were excised with sterile scalpel into small pieces of $1.0-1.5 \mathrm{~cm}$. The infected plant materials were sterilized with $3.85 \%$ sodium hypochlorite for $3 \mathrm{~min}$, rinsed in sterile distilled water three times. After drying the pieces on sterile blotting paper they were transferred to the Petri plates containing sterile filter papers $(9 \mathrm{~mm})$ and sterile distilled water. Incubation was done at $25 \pm 2^{\circ} \mathrm{C}$ for $6 \mathrm{~d}$ and after that the sporulated leaf pieces were transferred to potato dextrose agar (PDA) media. Incubation of sporulating leaf pieces at $12 \mathrm{~h}$ alternate light and darkness was done at $25^{\circ} \mathrm{C}$ for $6 \mathrm{~d}$ on PDA using the three methods of isolation mentioned early. Potato dextrose agar (PDA) medium was prepared according to Hussain et al., 2003. Sporulating lesions were shaken and the conidia were fallen on a clean glass slides and a drop of lactophenol stain was added. Samples were covered with cover slip and mounted on (100X and 400X magnifications) fitted with a camera. Fungal structures were observed. Conidial dimensions were measured. Conidial germination tests were carried out from infected leaves using camel hair brush and dropped onto PDA. The fungus was purified by single spore isolation technique (Hubballi et al., 2010).

Table 1. Scaling (0 to 3) of colony growth of Cryptosporiopsis sp and extent of contamination while using the three methods of isolation (direct conidial transfer, conidial suspension and transfer of lesion from advancing margin)

\begin{tabular}{lll}
\hline Scale & Growth & Contamination \\
\hline 0 & poor (no growth) & pure (non- contaminated) \\
& low growth $(<25 \%$ & less contaminated (e.g at corner or \\
1 & visible) & confined) \\
& Intermediate (26 upto & highly contaminated (but culturable, \\
2 & $60 \%$ visible) & confined) \\
3 & High $(>60 \%$ visible) & spoiled (discarded, not usable) \\
\hline
\end{tabular}

$10 \mathrm{ml}$ of clear, filtered $2 \%$ water agar was poured into sterilized Petri plates. Diluted spore suspension was prepared in sterile distilled water from 15-day-old culture. $1 \mathrm{ml}$ of such suspension was spread uniformly on agar plates. The plates were incubated at room temperature $\left(25 \pm 2^{\circ} \mathrm{C}\right)$ for $8 \mathrm{~h}$. The plates were examined under the microscope and germinated conidium was marked with ink on the surface of plate. The growing hyphal tip portion was transferred to PDA slants with the help of inoculation needle under aseptic conditions and was incubated at $25 \pm 2^{\circ} \mathrm{C}$ at room temperatures. Cultures were maintained on slants in sealed slants at room temperature (approx $25^{\circ} \mathrm{C}$ ). 


\subsection{Penetration and Infection Mechanisms of Cryptosporiopsis spp Causing Cashew Blight}

Leaflets on the other plants of the same age were marked and inoculated with a conidial suspension of $10^{6} \mathrm{ml}^{-1}$ on both sides. The inoculated plants were covered with plastic bags and kept for $10 \mathrm{~h}$ under high humidity (99\%) in the greenhouse. Inoculated leaves were stained with lactophenol. After every $4 \mathrm{~h}, 6 \mathrm{~mm}$ of the stained leaflet section was randomly cut with a cork borer and observed under a light microscope with high light intensity. Observations were made until symptom expression of leaves.

\subsection{Methods of Inoculating Cryptosporiopsis spp Causing Cashew Blight}

Three methods of inoculation, pin prick, clipping and paint brush were compared. Healthy leaves of cashew plants from a green house were excised at the petiole with sterile razor blade and covered immediately with a moistened sterile filter paper and transferred to the laboratory in polyethylene bags containing moistened filter paper. The leaves were washed in running tap water and rinsed in three changes of sterile distilled water and placed in Petri dishes lined with moist filter paper. The leaves were inoculated with suspension of fungal isolates containing $10^{6} \mathrm{cfu} / \mathrm{ml}$ using clipping method in which sterile scissors dipped in inoculum was used to cut $3 \mathrm{~cm}$ at leaves tip (Akhtar et al., 2008).

In pin prick method sterile pins dipped in inoculum were used to prick the leaves were on sides and in the center (Jabeen et al., 2011). In paint brush method the leaves were painted with inoculum. In control, the leaves were inoculated with sterile distilled water. 10 leaves were inoculated with $10^{6}$ spores $/ \mathrm{ml}$. Petri dishes were incubated at $25^{\circ} \mathrm{C}$ for six days and leaves observed daily for the development of symptoms. The lesion length was measured in $\mathrm{cm}$. The treatment was replicated thrice and data were analyzed statistically by analysis of variance (ANOVA) and significance at 5\% level was tests by Least Significant Difference (LSD). The fungus was re-isolated on PDA in pure culture and compared with the initial culture.

\subsection{Proving Pathogenicity of Cryptosporiopsis spp Causing Cashew Blight}

Cultures of Cryptosporiopsis spp (AA1, AA2, AA3, AA4, AA5, AA6, AA7, AA8, AAA9 and AA10) used in inoculation experiments were isolated in Tanzania and were selected for their capacity for good growth on PDA and profuse sporulation. Nine-month-old cashew AC 4 clone seedlings with three to six young tender leaves were spray-inoculated to run-off. A leaf-disk assay was developed for disease assessment of cashew leaf blight caused by Cryptosporiopsis spp (Olmstead and Lang, 2000). The second to fourth leaves below the apex were surface-disinfested by dipping entire leaves into $0.1 \%(\mathrm{v} / \mathrm{v})$ sodium hypochlorite three times and rinsed in sterile distilled water after each dip. Leaf disks (20-mm diameter) were then perforated from surface-disinfected leaves with a cork borer. The disks $(8$ per replication) were placed abaxial surface side up in Petri dishes containing a piece of 60 -mm-diameter sterilized filter paper. 2 $\mathrm{ml}$ of sterile water were added to the filter paper to maintain moisture. Spore suspension of cryptosporipsis spp was adjusted to $10^{6}$ spores per $\mathrm{ml}$ and was spot inoculated over the disks as inoculum, and then the disks were incubated in $25^{\circ} \mathrm{C}$. After $3 \mathrm{~d}$ of incubation, disease severity was assessed as percent necrotic area (Yousefi and Shahri, 2009).

Seeds of cashew were supplied by Naliendele Agricultural Research Institute were sown in soil-sand mixture enriched with N.P.K fertilizer in $2 \mathrm{~L}$ plastic pots. The pots were maintained in the greenhouse and were watered daily. After 3 weeks the seedlings were grafted using cashew AC 4 clone buds. Two month-old grafted cashew seedlings were used for pathogenicity test. Pathogenicity test was performed on 9 cashew seedlings by spraying conidial suspensions $\left(10^{6}\right.$ spores $\mathrm{mL}-1)$ of the isolates selected randomly on leaves of 9-month-old plants (Latiffah et al., 2009). Conidial suspensions were produced by growing mycelial mats of Cryptosporiopsis spp (AA1, AA2, AA3, AA4, AA5, AA6, AA7, AA8, AAA9 and AA10) on PDA in 9-cm Petri dishes for 10 days at $25^{\circ} \mathrm{C}$ in $12 \mathrm{hr}$ photoperiod and flooding with sterile distilled water containing a drop of Tween 80 . Concentrations of spore suspensions were assessed by using improved Neubaer Hemacytometer.

Inoculated plants and controls were enclosed in wet plastic bags for $48 \mathrm{~h}$. Control plants were sprayed with sterilized deionised water. After $48 \mathrm{~h}$ the plastic bags were removed and the plants observed daily for $10 \mathrm{~d}$. The symptoms were observed. The inoculated cashew seedlings were observed for symptom expression on the $1^{\text {st }}, 2^{\text {nd }}$ and $3^{\text {rd }}$ leaves of the seedlings. Koch's postulates were observed by consistently re-isolating the pathogen from the inoculated plants (Sharma et al., 2011). From the leaf lesions a Cryptosporiopsis species was re-isolated that was identical morphologically to the original isolate inoculated. Inoculations were repeated twice. Spores of Leaf and nut blight were taken from infected host tissue and mounted on a clean glass slide. Spores were mixed with lactophenol thoroughly in order to obtain a uniform spread, on which cover slip was placed. One hundred spores were measured under high power objective. The average size of the spore was calculated. Microphotographs were taken to show the typical spore morphology of the pathogen. Length and breadth of conidia were measured using a pre-calibrated ocular micrometer (Hosen et al., 2010)

\subsection{Pathogenicity Assays of Cryptosporiopsis spp Causing Cashew Blight on Pseudo-Fruits and Nuts}

Intact cashew apples and nuts were harvested at different maturation stages (3-10weeks) from cashew clone AC4 in experimental field plots of Naliendele, Mtwara, Tanzania and taken to the lab immediately for inoculation (Lopez and Lucas,. 2010a). Three whole apples and attached nuts of AC4 clone, in different stages of maturation (three per stage), were surface disinfected by consecutive immersion for $10 \mathrm{~min}$ in $3.85 \mathrm{~m} / \mathrm{v} \% \mathrm{NaOCl}$. The samples were rinsed in sterilized distilled water, dried with sterile tissue paper, placed inside 
plastic boxes $(30 \times 15 \times 10 \mathrm{~cm})$ lined with moist filter papers, and axially marked with black permanent ink. Drops $(10 \mathrm{~mL})$ of conidial suspension $\left(10^{6} \mathrm{~mL}-1\right)$ of isolate AA3 were placed at $1 \mathrm{~cm}$ intervals onto an area of $1.0 \mathrm{~cm}$ of the axial line of the surface of apples and nuts. The boxes were then closed and incubated at $25^{\circ} \mathrm{C}$, for $72 \mathrm{~h}$ in $12 \mathrm{~h}$ alternate light and darkness. Symptom types were evaluated $72 \mathrm{~h}$ after inoculation. The experiment (completely random design) was repeated three times and the data were transformed to for analysis of variance and comparison of the average scores by the LSD test $(\mathrm{P} \leq$ 0.05). Koch's postulates were observed by consistently re-isolating the pathogen from the inoculated nuts. From the nut lesions a Cryptosporiopsis species was re-isolated that was identical morphologically to the original isolate inoculated.

\subsection{Data Collection and Analyses}

Data from quantification of the radial growth, lesion length and methods of inoculation were analyzed using ANOVA (SAS, 2003) and further separation of means was done using Least Significant Difference at $\mathrm{P}<0.05$. Frequency counts and scale comparisons of the three isolation methods tested, the exact Wilcoxon test (Mehta and Patel, 2001) was used.

\section{Results}

The present investigation was carried out both in the laboratory and fields during 2010-11 in Southern Tanzania. The data was obtained at Naliendele Research Station, Mtwara $\left(040^{\circ} 09^{\prime} 15.61 "\right.$ E $10^{\circ} 22^{\prime} 40.98^{\prime \prime} \mathrm{S}, 141 \mathrm{M}$ a s 1,). Mtwara district rains vary from $935 \mathrm{~mm}$ to $116 \mathrm{~mm}$ in the hills and the plateau. Likewise temperatures vary from $27^{\circ} \mathrm{C}$ as the highest

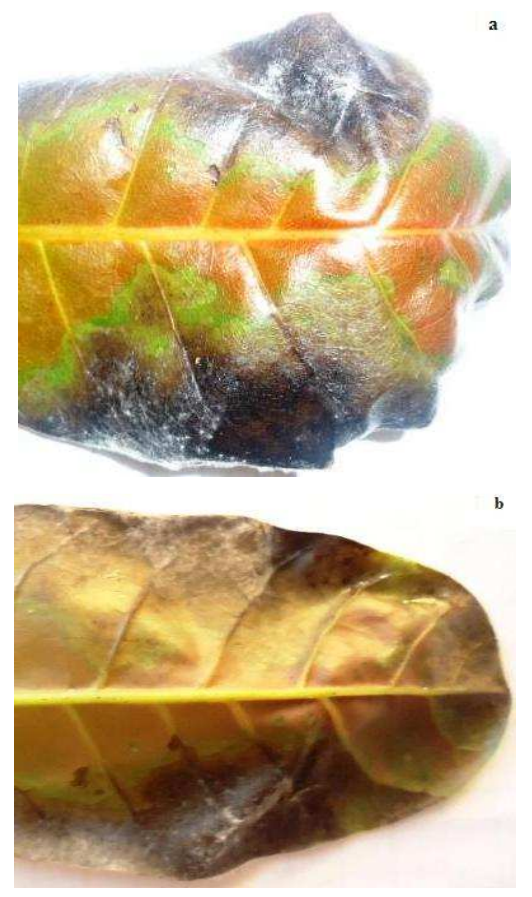

monthly mean at Mtwara on the coast in December to $23^{\circ} \mathrm{C}$ in July. Relative humidity goes from $87 \%$ in March to $79 \%$ in October in Mtwara. One isolate Cryptosporiopsis spp IMI 396316 for reference was obtained from CABI, UK.

\subsection{Macroscopic and Microscopic Observations of Symptoms and Signs of Leaf and Nut Blight}

Cashew leaf and nut blight infects young tender shoots, pseudo-fruits and nuts. Initially, the disease appears in the form of small, scattered brown spots on the leaf lamina. The abaxial surface of had more infections compared to adaxial part of young cashew leaves as observed. Later, these spots increase in size and coalesce covering larger leaf area (1 to 2 $\mathrm{cm}$ in diameter), with dark brown margin. Macroscopic symptoms of cashew blight caused by Cryptosporiopsis spp appeared as brown spots on both the upper (Fig 1a) and lower (Fig 1b) sides of cashew leaves. The first symptoms of the disease were chlorotic spots on the youngest tender leaves on both sides (Fig 1a and b). Scattered fruiting bodies are formed on affected leaves, young fruits and nuts. Leaf spots occurred on both sides of the leaves and varied in size, shape and color, within and between cashews. These include slightly depressed, pale grey to brown, with a dark-brown border, spreading necrotic lesions leading to a leaf blight symptom; circular, irregular or angular 1-2 cm in diameter; irregular roughened or corky lesions with eruption and necrosis of epidermal tissue, sometimes localized along veins. The diseased leaves curl and are defoliated (Fig. 1c). Generally, the symptoms coincided with flushing of shoots and flowering in the month of July to December. Cashew blight pathogen has an acervuloid conidiomata with discrete conidiogenous cells.
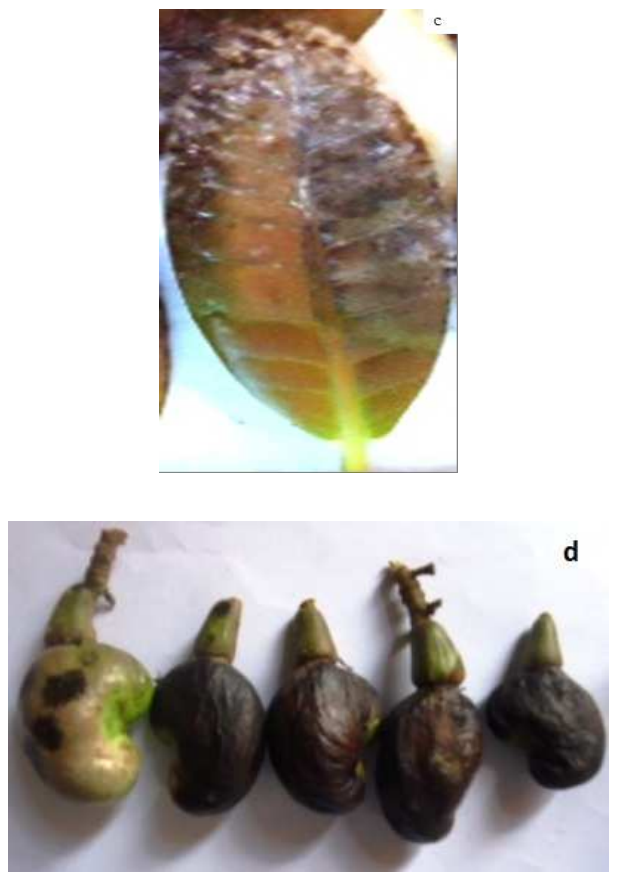

Fig. 1. Symptoms of leaf blight caused by Cryptosporiopsis sp on the adaxial (a) and abaxial (b) and c) leaf surface of Anarcadium occidentale L. (d) Infected cashew nuts 

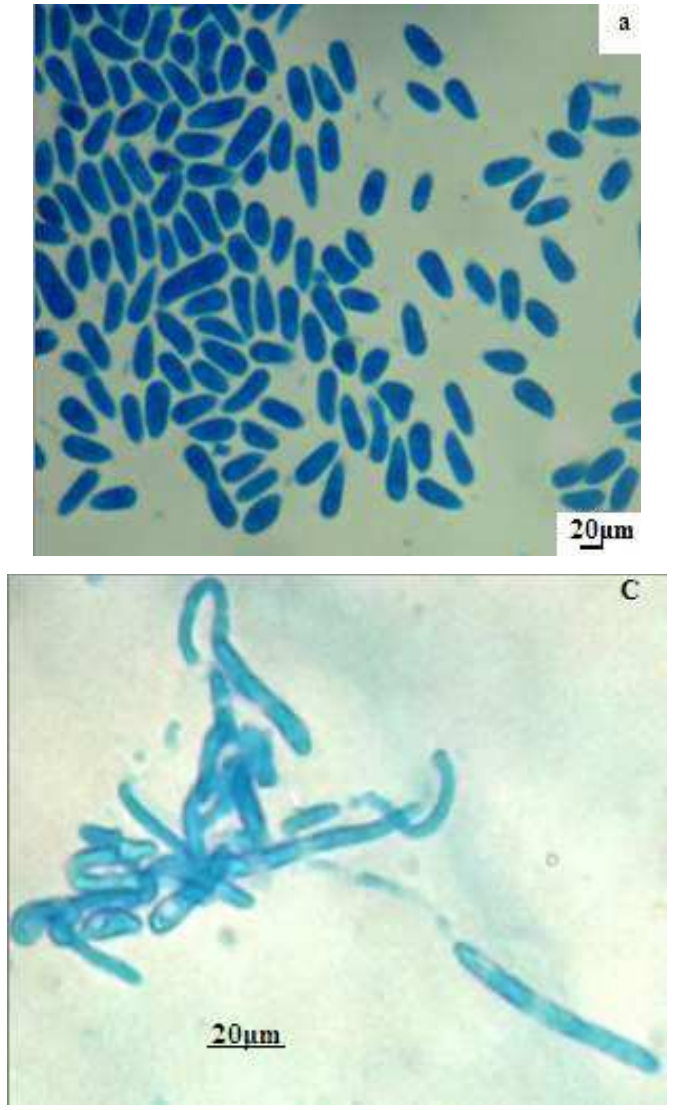
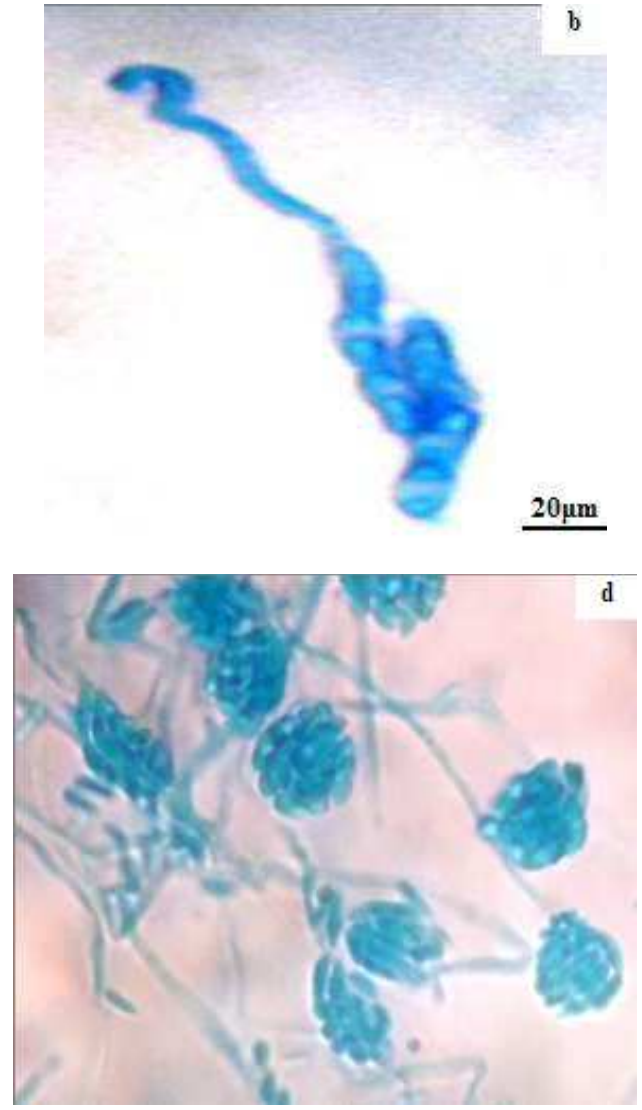

Fig. 2. lactophenol stained conidia (a) conidia (b and c) germinating conidia and conidiophores (d) of Cryptosporiopsis sp.

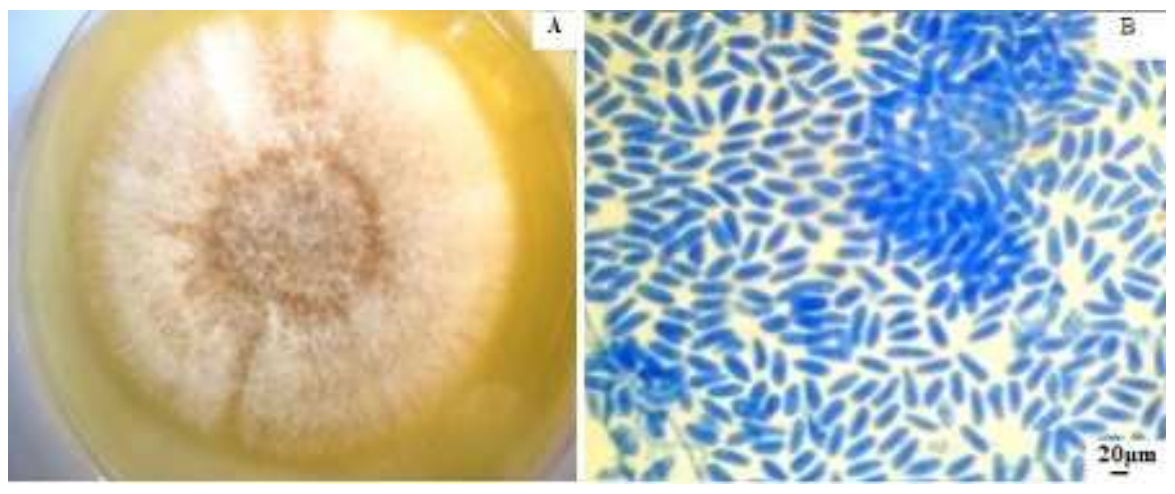

Fig. 3. (A) Cryptosporiopsis sp isolated on PDA (B) re-isolated Cryptosporiopsis sp conidia

Macroconidiogenous cells are discrete, phillidic and hyaline. Conidiomata were numerous over the surface and they are cream colored. Macroconidia were ellipsoidal, straight, aseptate, and arose from single phialides on vegetative hyphae or in conidiomata. Conidia stained with lactophenol solution and mounted on a microscope a minute later showed a better contrast (Fig 2a). Macroconidia are elongated and mostly one celled rounded at the apex, tapering abruptly into slightly protruding scar at the base (Fig 2a). Terminal germinating conidia were stained similarly after $10 \mathrm{~h}$ of inoculating slides containing the conidia at $27^{\circ} \mathrm{C}$. Macroconidia were thin walled brown to olive brown in color and are 0 -septate. Conidiomata develop on foliar lesions on blighted shoots. Branched conidiophores with sporulating conidia were observed.
Fruiting bodies were cup-shaped when moist with pigmented margins, bearing creamy masses of macroconidia (Fig. 2d). The conidiomata were scattered irregularly on lesions and erupt through the epidermis or stem periderm but can be quite inconspicuous when leaves are dry. Conidiospores phialides, formed in hemispherical sporodochial conidiomata (Fig 2d). They are borne terminally on simple conidiophores. Hyphae of young conidiomata are moniliform and hyaline, older conidiomata composed of black brown hyphae (Fig 2d). Conidiogenous cells solitary, cylindric, phialidic, $9-12 \times 12 \times 2-5 \mu \mathrm{m}$. Micronidia were not observed. Colonies of Cryptosporiopsis spp were white initially becoming pale to dark brown in age in PDA (Fig 3A). Macroconidia averaged $40.6 \pm 10.8 \mu \mathrm{m}$ in length and $10.7 \pm 2.3 \mu \mathrm{m}$ in width, with a 
length/width ratio of $4.0 \pm 1.4 \mu \mathrm{m}$ (Fig 3B).

\subsection{Isolation and Inoculation of Cryptosporiopsis spp Causing Cashew Blight}

Three methods of isolation were tested and direct conidial transfer proved to be the easiest and most efficient one. According to the ordinal scale used (0-3), isolation using direct conidial transfer produced less contamination and more growth, as compared to either conidial suspension or lesion transfer (Table 2). A similar result was recorded for the growth whereby growth of Cryptosporiopsis spp in direct conidial transfer proved to be significantly higher than in either conidial suspension or lesion transfer $(\mathrm{P}<0.05)$. Re-isolated fungi produced spores and conidiophores morphologically identical to those of the original field isolate used for inoculation when grown on PDA. In addition, when the fungus was re-isolated from symptomatic tissues results indicated cultures from experimentally inoculated seedlings were identical to that of the original isolate. Colonies on PDA were white to gray at the beginning and became orange with age. Conidial masses were white initially, becoming pale to golden yellow in age. The type culture of the fungus is maintained at the Naliendele Agricultural Research Institute, Tanzania. The pathogenic culture isolated from the diseased plants was identified on the basis of the morphological characters as Cryptosporiopsis spp and was confirmed from CABI, Plant clinic UK. Cashew leaves infected with blight were collected from 10 different parts of Southern Tanzania and 10 isolates of pure hyphal tip cultures of 10 isolates were maintained on PDA. Further, studies on cultural and morphological characters were carried out in the laboratory. The details of 10 different isolates are furnished below (Table 3 ). There were significant differences in the methods of inoculation used in the experiment $\mathrm{F}(2,9)=24.55, \mathrm{P}<.0001$. Inoculating using a paint brush recorded the highest mean lesion length $(1.2 \mathrm{~cm})$ followed by clipping method $(0.7842 \mathrm{~cm})$ and lastly pin pricking recorded the lowest mean lesion length (Table 4).

Table 2. Frequency count of $9 \mathrm{~cm}$ Petri dishes that were compared for growth and contamination of Cryptosporiopsis sp according to a rating of 0 to 3 after using three isolation methods (direct conidial transfer, conidial suspension transfer and lesion transfer).

\begin{tabular}{|c|c|c|c|c|c|c|c|c|c|c|}
\hline \multirow{2}{*}{ Method } & \multicolumn{5}{|c|}{ Contamination scale } & \multicolumn{5}{|c|}{ Growth scale } \\
\hline & 0 & 1 & 2 & 3 & significance & $\mathbf{0}$ & 1 & 2 & 3 & Significance \\
\hline Direct conidial transfer & 9 & 8 & 3 & 0 & $\mathrm{a}$ & 0 & 4 & 4 & 12 & $\mathrm{a}$ \\
\hline Conidial suspension transfer & 1 & 6 & 10 & 3 & $\mathrm{~b}$ & 6 & 5 & 9 & 0 & $\mathrm{~b}$ \\
\hline Lesion transfer & 2 & 12 & 6 & 0 & $\mathrm{c}$ & 0 & 13 & 7 & 0 & $\mathrm{c}$ \\
\hline
\end{tabular}

*Key for the scales are presented in Table 1

Table 3. Isolates obtained from sampled locations

\begin{tabular}{lll}
\hline Isolates & Infected organ & Place of origin \\
\hline AA1 & leaf & Naliendele \\
AA2 & leaf & Madangwa \\
AA3 & leaf & Mnazi mmoja \\
AA4 & pseudo fruit & Malamba \\
AA5 & pseudo fruit & Lyenje \\
AA6 & nut & Namiyonga \\
AA7 & nut & Chiola \\
AA8 & nut & Nandagala \\
AA9 & pseudo fruit & Nachingwea \\
AA10 & leaf & Newala \\
\hline
\end{tabular}

Table 4. Comparison of Cryptosporiopsis sp inoculation methods as measured by mean lesion length 6 days after inoculation on cashew leaves

\begin{tabular}{ll}
\hline Methods of inoculation & mean lesion length $(\mathbf{c m})$ \\
\hline paint brush & $1.2000 \pm 0.08^{\mathrm{a}}$ \\
clipping & $0.7482 \pm 0.05^{\mathrm{b}}$ \\
pin pricking & $0.6667 \pm 0.07^{\mathrm{c}}$ \\
CV\% & 34.567 \\
LSD & 0.1636 \\
\hline
\end{tabular}

\subsection{Penetration of Cryptosporiopsis spp Causing Cashew Blight}

Stomata of cashew leaves including the subsidiary and guard cells were easily visible on both leaf surfaces of the intact and cleared leaves from light microscopy (Fig 4). Stomatal penetration of Cryptosporiopsis spp was visible through the simple staining technique using lactophenol solution after $36-48 \mathrm{~h}$ of inoculation. Direct penetration of primary germ tube was seen through simple staining technique of the leaves. Penetration was by germinating conidia through a cuticle that caused an infection in leaves. Observation of the primary infection hyphae was observed after $6 \mathrm{~h}$ of inoculation on cashew leaves at the greenhouse (Fig 4). $12 \mathrm{~h}$ later, Secondary hyphae were observed growing sparsely in different directions. The appressorium was formed $12 \mathrm{~h}$ after inoculation and a layer of mycelium can be observed at $24 \mathrm{~h}$. The infection hyphae developing from the appressorium penetrated host cuticle and leaves showed symptoms $24 \mathrm{~h}$ after inoculation. The fungus causes lesions on cashew leaves as it progresses. Dark brown spots appear on leaves enlarging with irregular dark brown margins. Microscopic observations of inoculated leaves at $5 \mathrm{~d}$ of inoculation showed the extent of enlargement of the conidia to be released soon and serve for dispersing the pathogen.
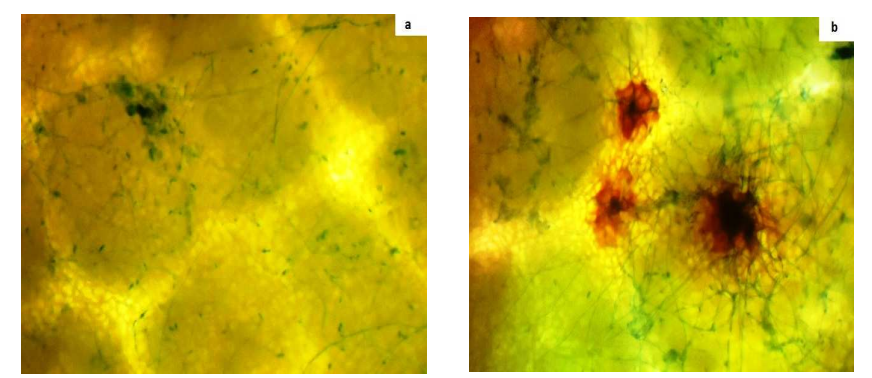

Fig. 4. Stomatal penetration of Cryptosporiopsis spp 


\subsection{Pathogenicity Cryptosporiopsis spp Causing Cashew Blight}

After $72 \mathrm{~h}$, cashew seedlings inoculated with conidial suspensions exhibited small brown spots on multiple leaves (Fig 5). Spots enlarged over time and closely resembled spots observed in the field, although disease severity appeared lower than for field plants. Among inoculated plants, $47.4 \pm$ 5.14 (mean $\pm \mathrm{SE}) \%$ of leaves displayed characteristic lesions, and the average disease severity per plant was $29.20 \%$. Sterile water control did not display any disease symptoms. After 72 h, leaves sprayed with Cryptosporiopsis spp isolate began curling thereafter to developed dark, irregularly shaped spots with black margins. The younger first leaves of cashew seedlings were more susceptible than the older second leaves (Fig 5). At this time, the fungus had grown through the leaves after spraying, and mycelium could be seen on the bottom of inoculated leaves. After 10 days, infected leaves were completely dead. Tufts of fungal mycelia could be observed emerging from infected leaves. In addition, leaves adjacent to the inoculation site began to display spots and lesions similar to those on inoculated plants in the pathogenicity assay. Young tender shoots were more susceptible to infection than older cashew leaves. Spores and conidiophores characteristic of a Cryptosporiopsis spp were prolific on lesions of most diseased leaves. Fungal spores were produced on brown to dark brown and aseptate conidiophores. There were significant differences in disease severity that was assessed as percent necrotic area among the sampled isolates, $\mathrm{F}(9,29)=263.2, \mathrm{P}<.0001$. Using leaf disc assay (Table 5). Isolate AA2 produced the highest mean percentage necrotic area $(49.29 \%)$ followed by isolate AA4 (49.18\%) and then AA7 (39.925\%). Isolate AA3 had the least mean percentage necrotic area (13.11\%). Infection of young nuts at stage 1 showed blackening. Infection of older nuts stage II and III resulted in darkening with white spore masses of the fungus were observed within the nut lesions (Fig 6). Ripened cashew apples (10 weeks) of AC 4 cashew clone showed early and many symptoms than immature cashew apples ( 3 weeks) showed fewer symptoms as observed. These could be attributed to presence of physical and chemical barriers. Developed nuts (10 weeks) showed no symptoms to both isolates while immature nuts (3weeks) exhibited symptoms, probably due to their thicker exocarp cuticle and reduced number of stomatal pores.

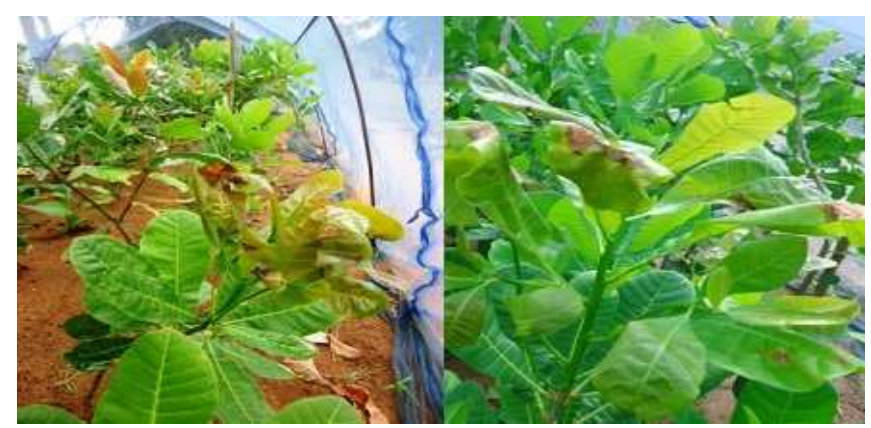

Fig. 5. Pathogenicity test on cashew clone AC 4 seedlings

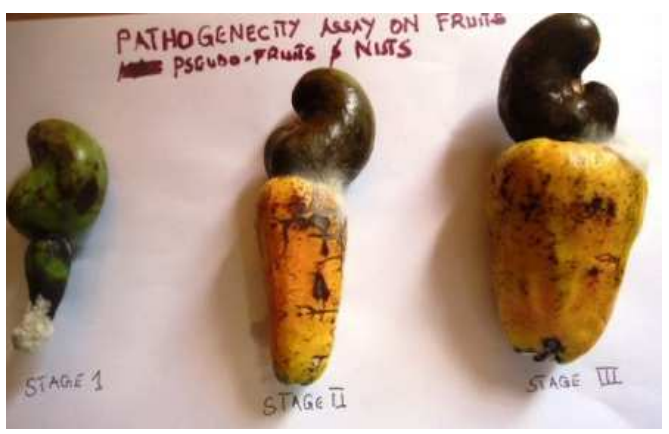

Fig. 6. Pathogenicity Assays on Pseudo-fruits and nuts

Table 5. percentage necrotic area of the isolates

\begin{tabular}{ll}
\hline Isolates & Mean percentage necrotic area \\
\hline AA1 & $14.606 \pm 0.52^{\mathrm{e}}$ \\
AA2 & $49.296 \pm 1.05^{\mathrm{a}}$ \\
AA3 & $13.110 \pm 0.88^{\mathrm{e}}$ \\
AA4 & $49.182 \pm 0.200^{\mathrm{a}}$ \\
AA5 & $19.983 \pm 1.312^{\mathrm{d}}$ \\
AA6 & $20.625 \pm 1.600^{\mathrm{d}}$ \\
AA7 & $39.925 \pm 0.339^{\mathrm{b}}$ \\
AA8 & $38.017 \pm 0.185^{\mathrm{b}}$ \\
AA9 & $19.624 \pm 0.748^{\mathrm{d}}$ \\
AA10 & $27.703 \pm 0.272^{\mathrm{c}}$ \\
CV\% & 5.0536 \\
LSD & 2.5139 \\
\hline
\end{tabular}

\section{Discussion}

Macroscopic observations of symptoms and signs of leaf and nut blight from natural and artificial inoculations confirmed many of earlier research outputs. General initial symptoms produced were spot lesions, with a brown margin, formed on leaves especially on the young tender leaves. Lesions caused by Cryptosporiopsis spp ranged from 1-30 $\mathrm{mm}$ in length and enlarged within a few days. The symptoms observed confirmed an earlier report by Sijaona et al., 2006 that described dark tan lesions on infected leaves. Symptoms of blight produced by the artificially inoculated and diseased plants were identical and confirmed with those symptoms observed on naturally infected cashew plants in the field. The pathogen has fruiting bodies that are irregularly scattered on affected leaves, nuts and fruits. Cryptosporiopsis spp causing cashew blight appears as leaf spots on living young leaves, pseudo-fruits and nuts

Macroconidia of cashew leaf and nut blight are typical of Cryptosporiopsis having ellipsoidal, rounded at the apex, tapering into a slightly protruding scar at the base. The scar at the base has been observed in six species of Cryptosporiopsis. Basic microscopic conidial morphology of Cryptosporiopsis spp causing cashew blight from this research fitted the range of morphological variations accepted in literature. Microscope inspection revealed that macroconidia formed in a group and were ellipsoidal, 0 -septate. The cashew blight macroconidia are relatively big in size $(40.6 \pm 10.8 \mu \mathrm{m}$ in length and $10.7 \pm$ $2.3 \mu \mathrm{m}$ in width) with other Cryptosporiopsis species including Cryptosporiopsis melanogena Kowalski and Halmschlager that has conidia measuring $21-34 \times 7.4-8.0 \mu \mathrm{m}$. Major variations in the conidial length and width among 
various species could be attributed to the prevailing weather conditions during mounting. Relative humidity in the study site ranges from $87 \%$ in March to $79 \%$ in October which might be attributed to the elongation of the conidia. These results are in agreement with Webster and Weber, 2007 findings that revealed excessive humidity caused elongation of conidia. Time duration between sample preparation and microscopic observation could also affect the conidial measurements.

The presence of vegetative hyphae in the cashew leaf and nut blight pathogen is similar to the $C$. radicicola Kowalski and Bartnik and C. melanogena Kowalski and Halmschhlager species. By its morphological and cultural characteristics, this fungus was identified as Cryptosporiopsis spp attacking cashew leaf and nut blight (Sijaona et al., 2006). The abaxial cashew leaf surface was used for inoculation in leaf disk assay because it was more susceptible than the adaxial one according to the macroscopic observations in the field. This confirms earlier report suggested the use of abaxial leaf surfaces, which are more susceptible than the adaxial ones and through which natural infections more frequently occur (Akaza et al., 2009). The isolates (AA1, AA2, AA3, AA4, AA5, AA6, AA7, AA8, AAA9 and AA10) from different sources of cashew parts showed different percentage necrotic area. This could have been attributed to their differences in virulence. However the inadequacy of morphological characterization in revealing special differences among the isolates calls for use of molecular characterization (Samiyeh et al., 2011).

Cashew blight pathogen has an acervuloid conidiomata similar to Cryptosporiopsis eucalypti Sankaran and B. Sutton and Cryptosporiopsis tarraconensis Gene and Guarro. Cryptosporiopsis spp causing cashew blight can be easily distinguished from other species in the genus because of the relatively big ellipsoid conidia. The pathogen can be further distinguished from other species in the genus based on its habit. There are a few morphological differences among the Cryptosporiopsis species and Cryptosporiopsis spp causing cashew blight. Six species of Cryptosporiopsis have thus far been reported from the roots of woody plants (Verkley et al., 2003). Cryptosporiopsis spp causing cashew blight was capable of penetrating epidermal cells of cashew leaves. The hyphae were sparse and grew in random directions. Fungi entered the host by direct penetration of the cuticle. However, the attraction of Cryptosporiopsis spp hyphae to the host surface was not established in this study.

Three methods of inoculation clipping, pin prick and paint brush were tested on detached leaves in vitro because detached leaves are accurate and biologically safe. The three methods of inoculation produced Cryptosporiopsis spp infections and symptoms of developing disease under laboratory conditions. There were differences in disease progression and final disease severity in the three methods. Clipping method of inoculation had high and similar disease progression rates with paint brush method, but the disease progression curve started earlier in plants inoculated with the clipping method, probably due to direct contact of the fungal suspension with the interveinal region of a leaf. Inoculation by pricking method using a pin impregnated with the fungal suspension produced the lowest lesion length levels. The delay between wounding and fungal inoculation can affect the ability of fungi to infect wounded tissues, since plant defenses are quickly induced in injured tissues (Venisse et al., 2002). Paint brush method of inoculation was found to be more effective as compared to other two methods based on the lesion size. Probably due to the increased leaf area that initially interacts with the pathogen inoculum.

Clipping method where the leaves were grasped and clipped by a pair of scissor dipped in fungal suspension was the better of the three methods probably because this approach assures direct access of the pathogen to the plant vascular system, and, compared to the other pin pricking, fewer fungal cells are needed to reach to start an infection. The cut ends provide entry for the pathogen and might be one of the main causes for spreading blight disease in cashew. The cell sap coming from injured tissue due to pin pricking usually serves as nutrient for microorganisms but this seemed not to be the case in Cryptosporiopsis spp causing blight.

The direct transfer of conidia or conidiophores from the underside of the infected leaves to Petri dishes containing PDA using a sterile scalpel proved to be the quickest, easiest and most effective way to isolate the fungus. The symptoms observed after inoculation of healthy cashew leaves with conidia of Cryptosporiopsis spp causing cashew blight is consistent with earlier reports (Sijaona et al., 2006). After incubation for 3 days, cashew leaves showed typical symptoms of leaf blight caused Cryptosporiopsis spp. Lesions coalesced and leaves curled was similar to earlier report by Sijaona et al., 2006. Fully expanded, mature leaves were immune to blight infection this could be attributed to the tough texture of these leaves. The tougher texture contained in older mature cashew leaves makes it more resistant for the pathogen entry. The number of trichomes on the adaxial surface of the mature cashew leaves is more compared to the young tender leaves making them more susceptible to Cryptosporiopsis spp causing cashew blight (Lopez and Lucas,. 2010b).

The abaxial surface of had more infections compared to adaxial part of young cashew leaves as observed this is due to the higher number of stomata and trichomes than the adaxial surface which might suggest easier fungal penetration and infection (Lopez and Lucas,. 2010a). Re-isolation from these leaves on PDA produced mycelia and conidia having same characteristics as the fungus inoculated confirming to its identification. Colonies on PDA were white to gray at the beginning and became orange with age. Young seedlings at the greenhouse confirmed the same symptoms after $10 \mathrm{~d}$. The pathogen was successfully re-isolated from the infected leaves and a pure culture of Cryptosporiopsis spp was obtained. Conidiomata of Cryptosporiopsis spp causing cashew blight on natural substrates was acervular, separate, with outer walls formed of pale brown to hyaline and thin-walled. Developed nuts showed no symptoms to both inoculations while immature nuts exhibited symptoms, probably due to their thicker exocarp cuticle and reduced number of stomatal pores. 


\section{Conclusion}

Basic microscopic conidial morphology of Cryptosporiopsis spp causing cashew blight from this research fitted the range of morphological variations accepted in literature. Cryptosporiopsis spp causing cashew blight was observed to have considerably slow growth to other fungal species therefore direct conidial transfer proved efficient. There is much still to learn regarding the biology of Cryptosporiopsis spp causing cashew blight, including its relationship to other Cryptosporiopsis species on different hosts. Biology and infection mechanisms studied here create bases for the morphological and molecular characterization of leaf and nut blight pathogen for better understanding of its biology, identification and classfication.

\section{Abbreviations}

$\begin{array}{ll}\text { GPC } & \text { Global Plant Clinic } \\ \mathrm{ml} & \text { milliliter } \\ \mathrm{mm} & \text { millimeter } \\ { }^{\circ} \mathrm{C} & \text { degrees centigrade } \\ \mathrm{ml}^{-1} & \text { milliliter per liter } \\ \text { ANOVA } & \text { analysis of variance } \\ \text { a.s.l } & \text { above sea level } \\ \mathrm{cm} & \text { centimeter } \\ \mathrm{N} . P . K & \text { Nitrogen Phosphorus Potassium } \\ \text { L } & \text { liter } \\ \text { v/v } & \text { volume per volume } \\ \text { LSD } & \text { Least Significant Difference } \\ \mathrm{min} & \text { minutes } \\ \mathrm{cfu} / \mathrm{ml} & \text { colony forming units per milliliter } \\ \mathrm{SAS} & \text { Statistical Analysis System } \\ \mathrm{d} & \text { days } \\ \mu \mathrm{m} & \text { micrometer }\end{array}$

\section{Acknowledgements}

We thank the German Federal Ministry for Economic Co-operation and Development (BMZ) for funding the cashew project, the International Centre of Insect Pathology and Ecology (ICIPE), Ministry of Agriculture and Food Security in Tanzania through Naliendele Agricultural Research Institute (NARI). We are grateful to CABI, UK, for providing a reference specimen.

\section{References}

[1] Adeniyi, D.O., Orisajo, S.B. Fademi, O.A. Adenuga, O.O. and Dongo, L.N. 2011. Physiological studies of fungi complexes associated with cashew diseases. J. Agric. Biol. Sci., 6: 34-38

[2] Akaza M. J., Goran J. A. K. N’, 'Guetta S-P.A. N, Kebe B. I., Tahi G.M. and Sangare A., (2009). Resistance to Phytophthora palmivora (Butler) Butler Assessed on Leaf Discs of Cacao
(Theobroma cacao L.) Hybrid Trees. Asian Journal of Plant Pathology, 3: 106-118.

[3] Akhtar, M. A. Abdul R. and Abdul H., 2008. Comparison of Methods of Inoculation of Xanthomonas Oryzae Pv.Oryzae in Rice Cultivars. Pak. J. Bot., 40(5): 2171-2175.

[4] Akinwale, T. O. 2000. Cashew apple juice: Its use in fortifying the nutritional quality of some tropical fruits. Eur. Food Res. Technol. 211: 205-207.

[5] Ammar M. I. and El-Naggar, M. A. (2011). Date Palm (Phoenix dactylifera L.) Fungal Diseases in Najran, Saudi Arabia. International Journal of Plant Pathology, 2: 126-135.

[6] Azam-Ali, S. and Judge, E. 2000. The global cashew industry-opportunities for small-scale processors. In: Proceedings of the international workshop on cashew production and processing-Cashing in on Cashew pp. 1-10. Marawila: Sri Lanka.

[7] Cavalcante, A. A. M., Rubensam, G. Picada, J.N. Da Silva, E.G. Moreira, J.C.F. and Henriques, J.A.P. 2003. Mutagenic evaluation, antioxidant potential and antimutagenic activity against hydrogen peroxide of cashew $(<\mathrm{i}>$ Anacardium occidentale $</ i>$ L.) apple juice and cajuina. Environ. Mol. Mut., 41: 360-369.

[8] Global Plant Clinic 2010. "Plant Doctors" a Global Prescription for Plant Pests. Volume 90. www.cabi.org/Uploads/File/.../Sarah/Issues\%20-\%20biosecuri ty.pdf.

[9] Hosen, M. I., Ahmed, A. U. and Islam, M. R. 2010. Variability and Pathogenicity in Bangladeshi Isolates of Botrytis cinerea Causing Botrytis Gray Mold in Chickpea (Cicer arietinum L.). Plant Pathology Journal, 9: 129-134.

[10] Hubballi, M., Nakkeeran, S. Raguchander, T. Anand T. and Samiyappan, R. 2010. Effect of Environmental Conditions on Growth of Alternaria alternata Causing Leaf Blight of Noni. World Journal of Agricultural Sciences 6 (2): 171-177.

[11] Hussain, A., Iqbal, S.M. Ayub N. and Haqqani,. A.M. 2003. Physiological Study of Sclerotium rolfsii Sacc.. Plant Pathology Journal, 2: 102-106.

[12] Jabeen, R., S. Ur-Rahman and A. Rais,. 2011. Evaluating BLB Resistance / Aggressivness in Rice through Best Inoculum Concentration, Inoculation and Application Methods. Pak. J. Bot., 43(5): 2635-2638.

[13] Kannan, V. R., Sumathi, C. S., Balasubramanian V. and Ramesh,. N. 2009. Elementary Chemical Profiling and Antifungal Properties of Cashew (Anacardium occidentale L.) Nuts. Botany Research International 2 (4): 253-257.

[14] Latiffah Z., Hayati, M.Z. Baharuddin S. and Maziah Z., 2009. Identification and Pathogenicity of Fusarium Species Associated with Root Rot and Stem Rot of Dendrobium. Asian Journal of Plant Pathology, 3: 14-21.

[15] Lopez, A. M. Q. and Lucas, J.A. 2010a. Reaction of dwarf cashew clones to Colletotrichum gloeosporioides isolates in controlled environment. Sci. Agric. (Piracicaba, Braz.), v.67, n.2, p.228-235.

[16] Lopez, A. M. Q. and. Lucas,. J. A. 2010b. Reaction of dwarf cashew clones to Colletotrichum gloeosporioides isolates in controlled environment. Sci Agric. (Piracicaba, Braz.), V.67,n.2. 
[17] Marc, A., Achille, T. F. Mory, G. Koffi, P.V.N. Georges,. A.N. 2011. Minerals composition of the cashew apple juice (Anacardium occidentale L.) of Yamoussoukro, Cote D'ivoire. Pakistan Journal of Nutrition 10(12): 1109-1114.

[18] Mehta, C. R. and Patel, N. R. 2001. StatXact-5 for windows. Manual, Cytel Software Cooperation, Cambridge, USA.

[19] Ogunwolu, S. O., Henshaw, F. O. Mock H.P. and Matro., A. 2010. Production of protein concentrate and isolate from cashew (Anacardium occidentale L.) nut. African Journal of Food Agriculture Nutrition and Development.

[20] Olmstead, J.W. and G.A. Lang, 2000. A Leaf Disk Assay for Screening Sweet Cherry Genotypes for Susceptibility to Powdery Mildew. Hortscience 35(2):274-277.

[21] Samiyeh R, Adam P. and Sijam, K. 2011. First Report of Diaporthe/Phomopsis Complex Isolates in Soybean from Malaysia and Their Longevity in Stored Seeds. Asian Journal of Plant Pathology, 5: 146-154.

[22] SAS Institute Inc 2003. SAS/STAT User's Guide. Version 9.1, SAS Institute Inc. Cary, North Carolina, USA.

[23] Sharma, P., Singh N. and Verma, O.P. (2011). First Report of Curvularia lunata Associated with Leaf Spot of Amaranthus spinosus. Asian Journal of Plant Pathology, 5: 100-101.
[24] Sijaona, M. E. R., Reeder, R. H. and Waller, J. M. 2006. Cashew leaf and nut blight - A new disease of cashew in Tanzania caused by Cryptosporiopsis sp. Plant Pathology 55 576.

[25] Umeh, J. C., 2007. Economic potentials of cashew. Proceedings of the Repositioning Cashew for Economic Growth and Development, July 10-11, 2007, Confluence Beach Hotel, Lokoja, Kogi State, Nigeria.

[26] Venisse, J. S., Malnoy, M. Faize, M. Paulin, J. P. and Brisset, M N. 2002. Modulation of defense responses of Malus spp. during compatible and incompatible interactions with Erwinia amylovora. Mol Plant Microbe Interact 15:1204-1212.

[27] Verkley, G. J., Zijlstra, J. D. Summerbell, R. C. and Berendse, F. 2003. Phylogeny and taxonomy of root-inhabiting $<\mathrm{i}>$ Cryptosporiopsis species $</ \mathrm{i}>$ and $<\mathrm{i}>\mathrm{C}$. rhizophila $</ \mathrm{i}>\mathrm{sp}$. nov., a fungus inhabiting roots of several $<\mathrm{i}>$ Ericaceae $</ \mathrm{i}>$. Mycol. Res.,107: 689-698.

[28] Webster, J. and Weber, R. 2007. Introduction to Fungi. $3^{\text {rd }}$ Edn., Cambridge University Press, Cambridge, UK., Pages: 841.

[29] Yousefi, A. and Shahri, M. H. 2009. Brown Spot Disease of Peach and Apricot Trees, Pathogenicity and Overwinter. Asian Journal of Plant Pathology, 3: 61-69. 